\title{
Cryo-FIB Milling and Lift-Out for Preparation of Specimens for Cryo-TEM
}

\author{
Michael J. Zachman ${ }^{1}$, Jade M. Noble ${ }^{2}$, and Lena F. Kourkoutis ${ }^{1,3}$
}

1. School of Applied and Engineering Physics, Cornell University, Ithaca, NY, USA.

2. R. F. Smith School of Chemical and Biomolecular Engineering, Cornell, Ithaca, NY, USA.

3. Kavli Institute for Nanoscale Science, Cornell University, Ithaca, NY, USA.

Cryo-TEM is a well-established technique for investigating the near-native structure of thin frozenhydrated specimens. Specimens too thick for direct imaging, such as whole cells or tissues, must, however, be thinned prior to analysis in the cryo-TEM. Traditionally, cryo-ultramicrotomy served this function by slicing thin sections from larger samples using a diamond blade. This physical cutting process can, however, induce sectioning artifacts [2]. In this talk, we will discuss cryo-focused ion beam (cryo-FIB) techniques, which were developed more recently as alternative thinning methods.

Traditional FIB lift-out at room temperature is routinely used for site-specific preparation of highquality TEM samples from bulk materials and devices. In this method, a focused beam of ions is used to remove material around a site of interest, forming a cross-sectional slice, or lamella. The lamella is then extracted from the bulk sample, transferred to a TEM grid with a nanomanipulator, and subsequently thinned to electron transparency. Early attempts to use FIB milling for preparation of frozen-hydrated biological samples involved installation of a liquid nitrogen cooled stage, which enabled thinning of whole frozen cells and subsequent imaging of sub-cellular structures by cryo-TEM [3]. In contrast to the traditional FIB lift-out preparation, these cryo-TEM samples were prepared directly on the TEM grid by milling entirely through the frozen specimen at a shallow angle, leaving a thin lamella or wedge nearly parallel to the supporting grid (Figure 1). While variations of this "on-grid" cryo-FIB technique have been successfully applied to a range of cells $[3,4]$, it is limited to samples on the order of microns thick.

Larger samples may be too thick to mill entirely through or require longer milling times with increased beam currents, which can induce sample damage. For preparation of thick vitrified samples, the traditional FIB lift-out method has been adapted to maintain the sample temperature below the devitrification point throughout the process. The development of cooled nanomanipulators, in combination with existing cryo-stages, has enabled proof of concept demonstrations of cryo-FIB lift-out performed on various cryo-immobilized soft materials [5-7], as well as samples with buried solid-liquid interfaces [8]. The concept of the technique is illustrated in Figure 2. Cryo-FIB lift-out is a powerful technique that provides access to the internal structure of extended specimens, thereby dramatically extending the reach of cryo-TEM. Combined with spectroscopic mapping methods such as cryoSTEM/EDX or EELS, it enables researchers to study not only the structure but also the chemistry of buried structures at the nanometer scale (Figure $2 \mathrm{~g}$ ). The focus of this talk will be on practical aspects of cryo-FIB, including milling geometries and artifacts; how to obtain high-quality, uniform lamellas; and how to localize buried structures [9].

\section{References:}

[1] J. Dubochet et al, Q. Rev. Biophys. 212 (1988), p. 129.

[2] A. Al-Amoudi, D. J. Studer, and J. Dubochet, J. Struct. Biol. 150 (2005), p. 109.

[3] M. Marko, et al, Nat. Methods 4 (2007), p. 215. 
[4] E. Villa, et al, Curr. Op. Struct. Biol. 23 (2013), p. 771.

[5] S. Rubino et al, J. Struct. Biol. 180 (2012), p. 572.

[6] C.D.J. Parmenter, et al, Microsc. Res. Tech. 79 (2016), p. 298.

[7] J. Mahamid et al, J. Struct. Biol. 192 (2015), p. 262.

[8] M. Zachman et al, Microsc. Microanal. 22 (2016), p. 1338.

[9] This work was supported by NSF (DMR-1654596) and the Packard Foundation. It made use of the CCMR Shared Facilities which are supported through the NSF MRSEC program (DMR-1120296).
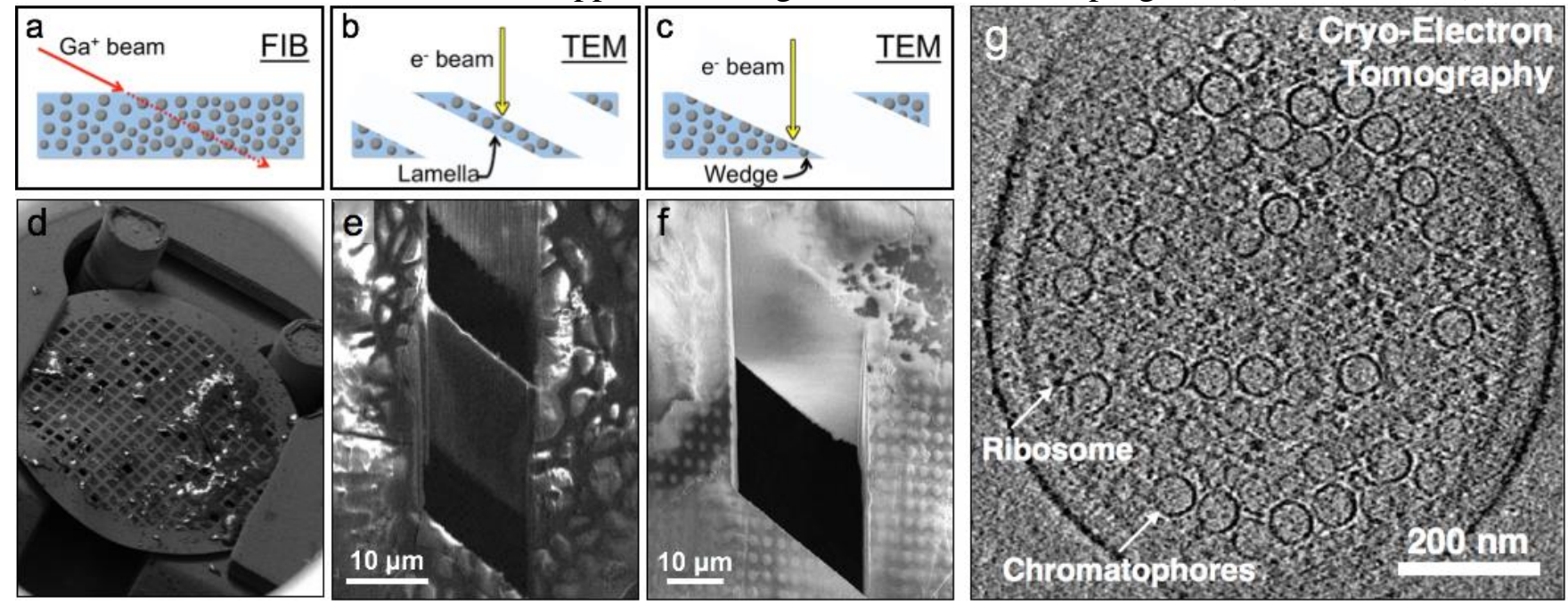

Figure 1. On-grid cryo-FIB thinning of specimens in preparation for cryo-TEM analysis. (a) A focused gallium ion beam is used for site-specific milling. This allows lamellas $(\mathbf{b}, \mathbf{e})$ and wedges $(\mathbf{c}, \mathbf{f})$ to be formed, producing regions thin enough for imaging in the TEM. A liquid nitrogen cooled sample stage enables thinning of vitrified samples directly on TEM grids (d), which can subsequently be loaded into the cryo-TEM for high-resolution characterization. Combined with cryo-electron tomography, cryo-FIB provides access to the 3D organization and architecture of macromolecules inside cells too thick for direct imaging, as illustrated for Rba. sphaeroides in (g).
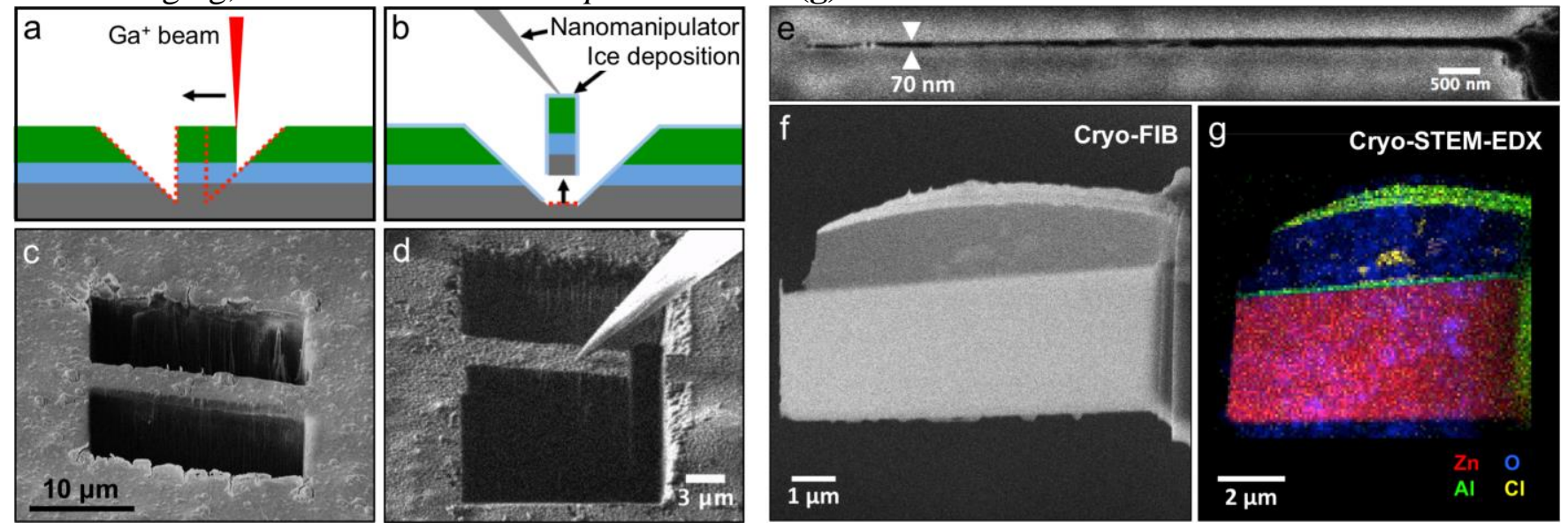

Figure 2. Preparation of electron transparent lamellas of frozen-hydrated specimens by cryo-FIB lift-out. (a, c) Site-specific milling of a lamella from the vitrified sample. (b, d) Attachment of the frozen lamella to a liquid nitrogen cooled nanomanipulator using water vapor. After similar attachment to a cooled TEM grid, the lamella is thinned to electron transparency. (e) A top-down image of a lamella created from a solid-liquid interface shows sample thinning to $<100 \mathrm{~nm}$. The final sample (f) was subsequently transferred to the cryo-STEM for nanoscale chemical analysis by EDX $(\mathbf{g})$. 